\title{
Factors associated with number of duodenal samples obtained in suspected celiac disease
}

\section{(ㄷ)(1) $\odot($}

\section{Authors}

Leonid Shamban ${ }^{1}$, Serge Sorser ${ }^{2}$, Stan Naydin ${ }^{3}$, Benjamin Lebwohl ${ }^{4}$, Mousa Shukr ${ }^{5}$, Charlotte Wiemann ${ }^{5}$, Daniel Yevsyukov6$^{6}$, Michael H. Piper ${ }^{7}$ Bradley Warren ${ }^{7}$, Peter H. R. Green ${ }^{8}$

Institutions

1 Gastroenterology, Genesys Regional Medical Center, Grand Blanc, Michigan, United States

2 Gastroenterology, Providence-Providence Park Hospital, Novi, Michigan, United States

3 Internal Medicine, Drexel University, Philadelphia, Pennsylvania, United States

4 Clinical Medicine, Columbia University, New York, New York, United States

5 Internal Medicine, Providence-Providence Park Hospital, Southfield, Michigan, United States

6 Division of Solid Organ Transplant, University of Minnesota Medical School Twin Cities, Minneapolis, Minnesota, United States

7 Gastroenterology, Providence-Providence Park, Southfield, Michigan, United States

8 Clinical Medicine, Columbia University, New York, New York, United States

submitted 30.12.2016

accepted after revision 26.6.2017

\section{Bibliography}

DOI https://doi.org/10.1055/s-0043-120522 |

Endoscopy International Open 2017; 05: E1220-E1228

(c) Georg Thieme Verlag KG Stuttgart · New York ISSN 2364-3722

\section{Corresponding author}

Leonid Shamban, DO, Gastroenterology, Genesys Regional Medical Center, One Genesys Parkway, Grand Blanc,

Michigan 48439-1477, United States

Fax: +1-810-606-5990

Ishamban@gmail.com

\section{ABSTRACT}

Background and study aims Many people with celiac disease are undiagnosed and there is evidence that insufficient duodenal samples may contribute to underdiagnosis. The aims of this study were to investigate whether more samples leads to a greater likelihood of a diagnosis of celiac disease and to elucidate factors that influence the number of samples collected.

Patients and methods We identified patients from two community hospitals who were undergoing duodenal biopsy for indications (as identified by International Classification of Diseases code) compatible with possible celiac disease. Three cohorts were evaluated: no celiac disease (NCD, normal villi), celiac disease (villous atrophy, Marsh score 3), and possible celiac disease (PCD, Marsh score <3). Endoscopic features, indication, setting, trainee presence, and patient demographic details were evaluated for their role in sample collection.

Results 5997 patients met the inclusion criteria. Patients with a final diagnosis of celiac disease had a median of 4 specimens collected. The percentage of patients diagnosed with celiac disease with one sample was $0.3 \%$ compared with $12.8 \%$ of those with six samples $(P=0.001)$. Patient factors that positively correlated with the number of samples collected were endoscopic features, demographic details, and indication $(P=0.001)$. Endoscopist factors that positively correlated with the number of samples collected were absence of a trainee, pediatric gastroenterologist, and outpatient setting $(P<0.001)$.

Conclusions Histological diagnosis of celiac disease significantly increased with six samples. Multiple factors influenced whether adequate biopsies were taken. Adherence to guidelines may increase the diagnosis rate of celiac disease.

\section{Introduction}

There is interest in factors associated with the underdiagnosis of celiac disease [1]. An accurate diagnosis relies on combining clinical, serological, and histological data, with a greater emphasis recently being placed on histological data [2]. Therefore, the final diagnostic component of celiac disease rests with the endoscopist and the pathologist.

To diagnose celiac disease, the most recent American College of Gastroenterology (ACG) guidelines recommend multiple biopsies of the small bowel: one or two biopsies of the bulb and at least four of the distal duodenum [3]. These guide- 
- Table 1 Pre-endoscopy International Classification of Diseases - ninth revision diagnosis codes.

\begin{tabular}{|c|c|c|c|}
\hline Indication & ICD-9 code & Grouping & Patients, $\mathrm{n}$ \\
\hline \multicolumn{4}{|l|}{ Abdominal pain, other specified site } \\
\hline Abdominal pain, unspecified site & 789.00 & 0 & 1600 \\
\hline \multicolumn{4}{|l|}{ Abdominal pain generalized } \\
\hline Anemia unspecified & 285.9 & & \\
\hline Iron deficiency anemia & 280.9 & 1 & 1294 \\
\hline Chronic blood loss anemia & 280.00 & & \\
\hline Nausea with vomiting & 780.71 & & \\
\hline Vomiting & 787.03 & 2 & 901 \\
\hline Nausea & 787.02 & & \\
\hline Diarrhea & 787.91 & 3 & 209 \\
\hline Epigastric abdominal pain & 789.06 & 4 & 1105 \\
\hline Abnormal weight loss & 783.21 & 5 & 204 \\
\hline Right upper quadrant abdominal pain & 789.01 & 6 & 128 \\
\hline Periumbilical abdominal pain & 789.05 & 7 & 16 \\
\hline Right lower quadrant abdominal pain & 789.03 & 8 & 19 \\
\hline Left upper quadrant abdominal pain & 789.02 & 9 & 33 \\
\hline Left lower quadrant abdominal pain & 789.04 & 10 & 23 \\
\hline Celiac disease (unconfirmed pre-endoscopy) & 579.0 & 11 & 13 \\
\hline Dyspepsia and other specified & 536.8 & 12 & 261 \\
\hline Other symptoms involving the digestive system & 787.99 & 13 & 178 \\
\hline Flatulence eructation and gas pain & 787.3 & 14 & 21 \\
\hline Transaminasemia & 790.14 & 15 & 0 \\
\hline
\end{tabular}

lines differ from those issued by the American Gastroenterological Association in 2006, which only recommended four to six samples from the distal duodenum [4]. Despite these recommendations, the sensitivity of duodenal biopsy may be decreased by the patchy distribution of disease [5], quality of the biopsy specimen [6], and the variability in pathological interpretation [7]. Biopsies are performed on patients because of abnormal serology, high index of suspicion, routine duodenal biopsy at endoscopy for disease indications other than celiac disease or endoscopic visualization of villous atrophy $[8,9]$. A recent survey revealed a discrepancy between the number of biopsies recommended and real-life practice, showing that $63 \%$ of patients had fewer than four duodenal biopsies [10].

In the current study, we investigated the yield of celiac disease diagnosis with respect to the number of samples collected. Subsequently, we evaluated patient and endoscopist factors that affected the number of samples obtained.

\section{Patients and methods}

Institutional Review board approval was obtained (404688-1). In this retrospective study, we reviewed the records from two community hospitals in Michigan, United States: St. John Providence Hospital and Medical Center, in Southfield and St. John Providence Park Hospital and Medical Center, in Novi, Michigan. Both inpatient and outpatient endoscopies were evaluated between 1 January 2008 and 11 February 2013. Patients were included in the study according to International Classification of Diseases - ninth revision (ICD-9) codes for endoscopy indications ( $>$ Table 1 ). The ICD-9 codes were selected according to all possible gastrointestinal manifestations of celiac disease, as defined by the ACG [3]. Patients who did not meet inclusion criteria were excluded ( $>$ Fig. 1). Patients were also excluded if there was a pre-endoscopy explanation for the ICD-9 codes chosen, and thus, a low pretest probability for celiac disease.

A total of 39 endoscopists were reviewed, three of whom were pediatric endoscopists. Their expertise ranged from 1 


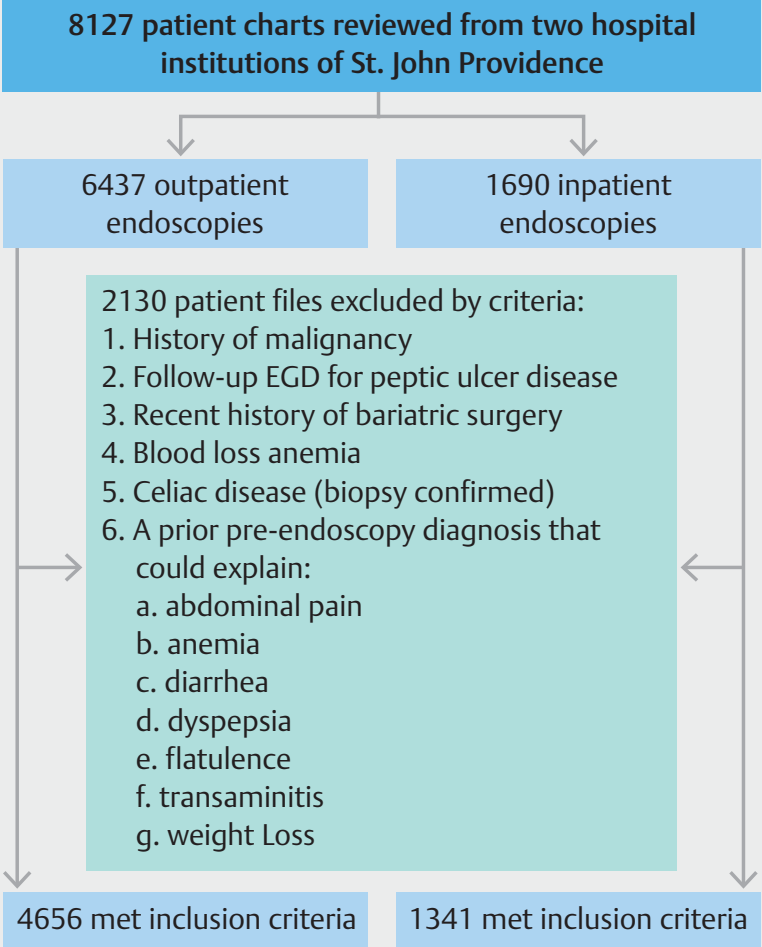

Fig. 1 Exclusion criteria. EGD, esophagogastroduodenoscopy.

year to more than 30 years of clinical practice. Trainees were from all 3 years of a fellowship program (three per year).

Monitored anesthesia care with deep propofol sedation was used in all adult and pediatric patients. Two types of gastroscopes were used in the adult population: Olympus GIF160 and GIF180 (Olympus, Tokyo, Japan). The Olympus GIF-XP160 gastroscope was used in pediatric patients. White-light endoscopy with a two-bite technique was used for all sample collections.

Endoscopic features were grouped into three categories: normal features with no gross pathology, classic celiac disease features, and all other appearances. Classic celiac disease features were findings in the endoscopy reports that were defined by previous studies by Lee and Green in 2005 [11]: loss or reduction in duodenal folds, mosaic mucosal pattern, scalloped configuration of the duodenal folds, micronodular pattern or increased vasculature pattern in the duodenum.

Pathological diagnoses by seven general pathologists, with more than 5 years of experience, were categorized as no celiac disease (NCD), celiac disease, or possible celiac disease (PCD). In cases of doubt, intradepartmental consultation was obtained. Celiac disease was defined according to the modified Marsh criteria [12]: shortening of villi and crypt hyperplasia with a villous to crypt ratio of less than $3: 1$, presence of intraepithelial lymphocytosis (IEL), more than 40 IELs. If lesions were less than the original Marsh-Oberhuber 3a classification [13], they were categorized as PCD; this group included Marsh I and II le- sions, and villous atrophy that did not fulfill Marsh criteria (villous atrophy without increased IELS).

\section{Statistics}

Statistical analysis was performed on the data using univariate, multivariate with one-way between-groups analysis of variance, $t$ tests, and chi-squared analysis using SPSS 15 (SPSS Inc., Chicago, Illinois, USA).

\section{Results}

A total of 8127 patients were reviewed and 5997 met the criteria for analysis ( $\triangleright$ Fig. 1 ). Patients were categorized into three cohorts as determined by pathology. Of the 5997 patients, 41 patients $(0.7 \%)$ were diagnosed with celiac disease and 62 $(1.0 \%)$ were classified as PCD ( $\bullet$ Table 2$)$. Of all patients, 4038 $(67.3 \%)$ were female and 5530 (92.2\%) were over the age of 20 years. Of the 4844 patients for whom race was documented, 2854 (58.9\%) were Caucasian. Endoscopies were performed in the outpatient setting in 4656 patients $(77.6 \%)$, and trainees were involved in 2045 procedures (34.1\%).

The frequency of celiac disease diagnosis increased with increasing sample number ( $\boldsymbol{\nabla}$ Fig. 2 ). Using chi-squared analysis, the prevalence of celiac disease diagnosis was $0.3 \%$ when fewer than four specimens were collected compared with $4.7 \%$ when four or more were collected (chi-squared $=139.7, P<0.001$ ). The prevalence of celiac disease diagnosis increased to $12.8 \%$ when six or more specimens were collected compared with $0.4 \%$ when fewer than six were collected (chi-squared= $304.22, P<0.001)$. The percentage of patients diagnosed with PCD from only one sample was $2.3 \%$ and peaked at $3.1 \%$ for four specimens.

A total of 508 patients ( $8.5 \%$ ) had four or more biopsies performed. Four or more specimens were obtained with increased frequency during the study, with 20 cases in 2008 and 126 cases in 2012. A similar pattern was seen for six biopsies, with 9 cases in 2008 and 44 cases in 2012. Of the patients diagnosed with celiac disease, $43.9 \%$ had six specimens collected, $9.8 \%$ had four biopsies, and $14.6 \%$ were diagnosed from two specimens. The likelihood of having a diagnosis of PCD was more common with two samples (43.5\%), with the diagnosis of PCD falling to $4.8 \%$ in patients with six samples.

Each variable was assessed for its number of biopsies in relation to final diagnosis. Supplemental $\triangleright$ Fig. 1 demonstrates the mean number of biopsy samples in relation to indication. Diagnoses for all types of ICD-9 abdominal pain codes were combined. Diarrhea for the celiac disease group had a mean of 5.0 specimens, whereas anemia had a mean of 4.0 samples. When celiac disease was suspected as the indication, a mean of 4.0 biopsies were performed. In the NCD and PCD cohorts, there was a statistically significant difference between the indications using an unpaired $t$ test $(P<0.001$ and $P=0.001$, respectively). Owing to the limited sample size, only indications for abdominal pain, anemia, and diarrhea were used in the PCD group for this analysis. No difference was observed in the celiac disease group $(P=0.59)$. 
- Table 2 Patient demographic details. Due to limited number of samples, patients with more than six biopsies have not been included.

\begin{tabular}{|c|c|c|c|}
\hline Diagnosis & NCD, no. of patients & Celiac disease, no. of patients & PCD, no. of patients \\
\hline \multicolumn{4}{|l|}{ Race, $\mathrm{n}$} \\
\hline - Caucasian & 2793 & 28 & 33 \\
\hline - African American & 1916 & 4 & 10 \\
\hline - Asian & 43 & 0 & 0 \\
\hline - Middle Eastern & 12 & 1 & 0 \\
\hline - Hispanic & 4 & 0 & 0 \\
\hline - Race other or unreported & 1126 & 8 & 19 \\
\hline \multicolumn{4}{|l|}{ Sex, n } \\
\hline - Female & 3965 & 34 & 39 \\
\hline - Male & 1929 & 7 & 23 \\
\hline Age, mean, years & 55 & 39 & 42 \\
\hline Patients $<20$ years, $n$ & 445 & 8 & 14 \\
\hline \multicolumn{4}{|l|}{ No. of biopsies, n (\%) } \\
\hline .0 & $3660(62.1)$ & $0(0)$ & $0(0)$ \\
\hline . 1 & $331(5.6)$ & $1(2.4)$ & $8(12.9)$ \\
\hline .2 & $975(16.5)$ & $6(14.6)$ & $27(43.5)$ \\
\hline .3 & 465 (7.9) & $10(24.4)$ & $15(24.1)$ \\
\hline . 4 & $243(4.1)$ & $4(9.8)$ & $8(12.9)$ \\
\hline .5 & 109 (1.8) & $2(4.9)$ & $1(1.6)$ \\
\hline .6 & $111(1.9)$ & 18 (43.9) & $3(4.8)$ \\
\hline Total diagnosis & 5894 & 41 & 62 \\
\hline
\end{tabular}

Analyzing the three groups for endoscopic features, patients with classic endoscopic celiac features had more samples collected ( $>$ Fig.3). Differences were seen in the NCD, PCD, and celiac disease cohorts for mean number of samples collected in relation to endoscopic features $(P=0.001, P=0.04, P=0.06$, respectively).

When endoscopy was performed in the inpatient setting, 5 out of 1341 patients $(0.4 \%)$ were diagnosed with celiac disease, and $16(1.2 \%)$ with PCD. A mean of 0.46 specimens were collected within the inpatient group compared with 1.17 in the outpatient setting $(P=0.001)$. No difference in mean specimen number was observed for celiac disease and PCD $(P=0.11$ and $P=0.48$, respectively).

When a pediatric gastroenterologist performed endoscopy (compared with an adult gastroenterologist), a greater median number of biopsies was obtained for all three cohorts. Only three pediatric gastroenterologists were reviewed, and 468/ 5997 patients were under the age of 18 years. Supplemental - Fig. 2 shows that pediatric patients with celiac disease had a narrower range of biopsy sample number compared with adult patients with celiac disease. Using an unpaired $t$ test, no difference was observed for pediatric patients in the celiac disease and PCD cohorts compared with the adult cohorts $(P=0.35$ and $P=0.63$, respectively). A difference was observed for the NCD cohort $(P<0.001)$.

With regard to patient demographic details and trainee presence, the three cohorts were aggregated before analysis was performed. With respect to sex, female patients had a statistically greater mean number of specimens collected 1.07 compared with 0.96 specimens collected for male patients $(P=0.004)$. Only 4844 patients identified their race: 2854 (58.9\%) were Caucasian, 1930 (39.8\%) were African American, $43(0.9 \%)$ were Asian, and 13 patients (0.3\%) were of MiddleEastern descent. Only Caucasian and African American patients were compared owing to the small number of patients in the other groups. Caucasian patients had a mean of 1.2 specimens collected compared with 0.6 for African American patients $(P<$ 0.001 ). With respect to age, mean biopsy number generally decreased with advancing age, and this decline was significant for the no celiac disease group $(P<0.001)(\triangleright$ Fig. 4$)$. In the presence of a trainee, on average fewer specimens were collected - 0.9 compared with 1.1 without a trainee $(P<0.001)$. 


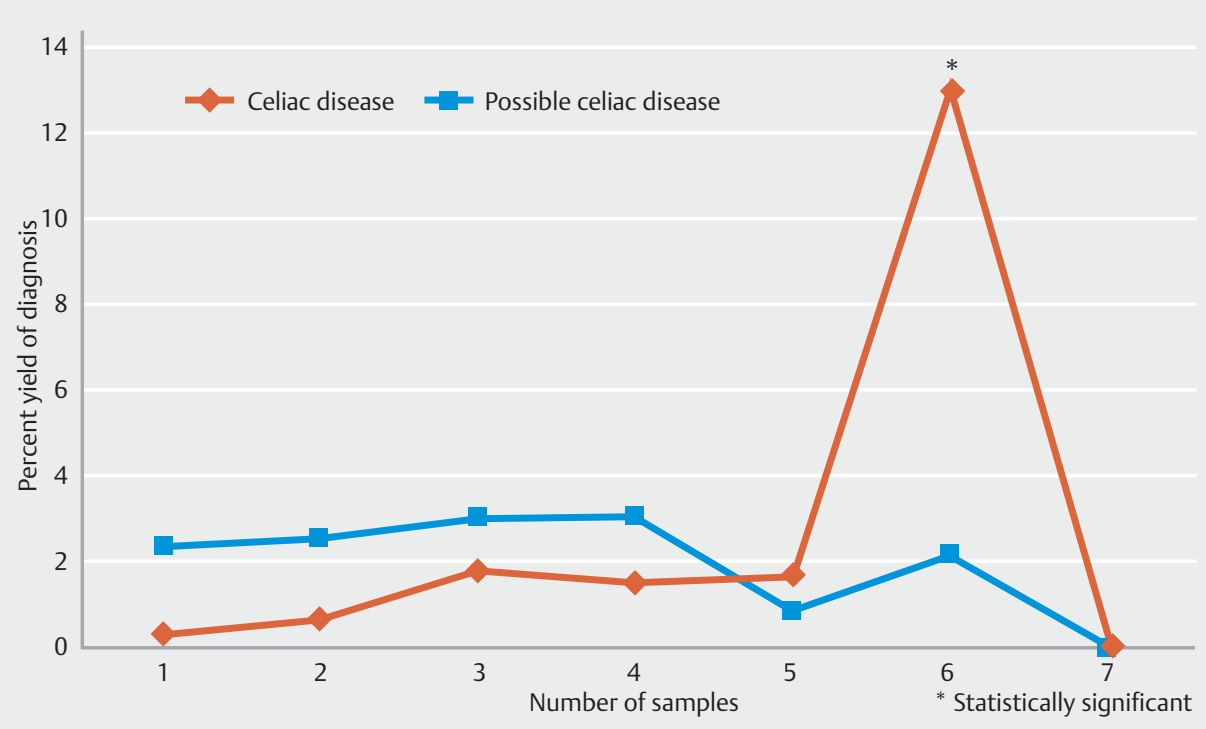

Fig. 2 Diagnostic yield based on number of biopsies.

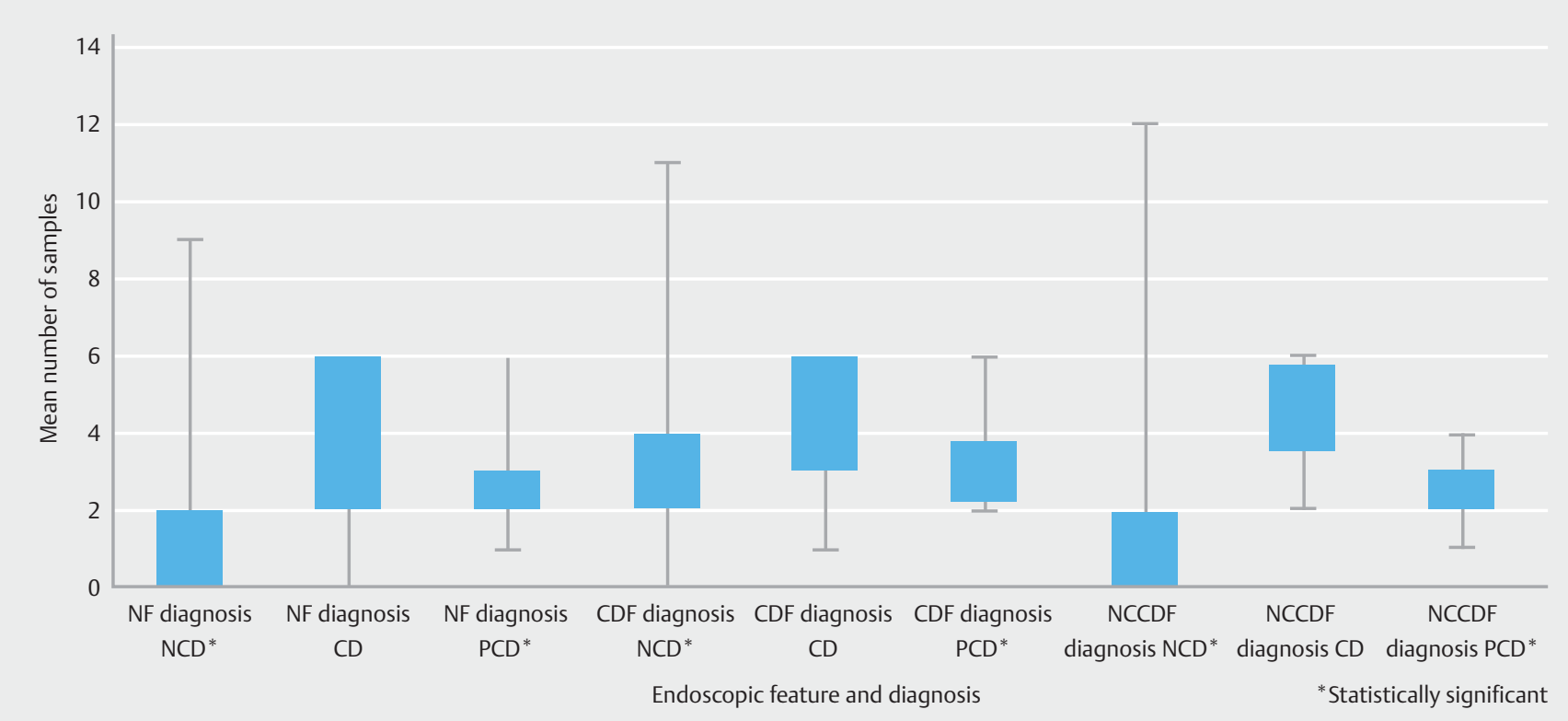

- Fig. 3 A box-whisker plot revealing the distribution of the mean number of samples collected for each diagnosis cohort and their respective endoscopic features. NF, normal features; CDF, classic disease features; NCCDF, nonclassic celiac disease features; PCD, possible celiac disease; CD, celiac disease; NCD, no celiac disease.

\section{Discussion}

The results of this study have confirmed previous studies demonstrating that celiac disease diagnosis is dependent on the number of biopsies taken. Patient factors, such as endoscopic features, indication, and demographic details, and endoscopist factors such as absence of a trainee, type of gastroenterologist (pediatric vs. adult), and the endoscopy setting (inpatient vs. outpatient) appear to determine whether an adequate number of biopsies are taken.
We saw a significant increase in celiac disease diagnosis when six or more samples were collected. This may be due to a small sample size; however, this observation was not noted with four and five specimens compared with two and three specimens as seen in previous studies [14]. Overall, 499 patients $(8.3 \%)$ with suspected celiac disease had four or more specimens obtained; however, the percentage of patients with six or more specimens submitted increased annually. Our results are comparable to previous studies that showed patients undergoing esophagogastroduodenoscopy (EGD) with celiac dis- 


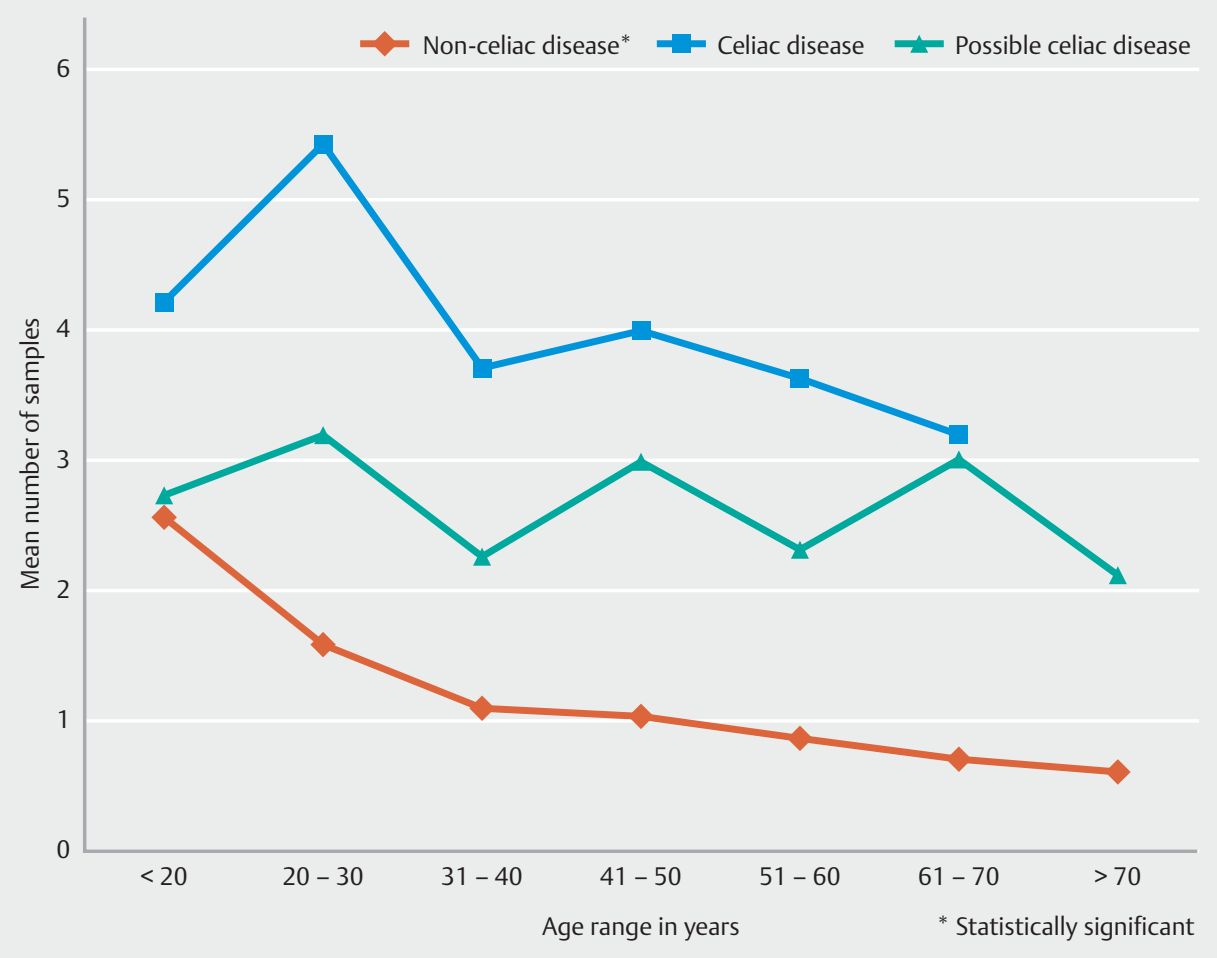

- Fig. 4 Mean number of biopsy samples by age.

ease as a differential diagnosis often do not undergo duodenal biopsy, nor are the recommended number of specimens collected $[14,15]$.

We found that patient factors that can influence whether an adequate number of biopsies are taken include endoscopic features and demographic details. Demographic details include the patient's age (pediatric vs. young adult vs. older adult), race, and sex. The mean number of biopsies increased for classic endoscopic features of celiac disease, for Caucasian patients vs. African American patients, for female vs. male patients, and for younger vs. older patients. Other patient factors include the indication for endoscopy. For classic celiac disease symptoms and manifestations, mean biopsy number was greater than for nonspecific celiac disease symptoms. Additionally, for endoscopist factors, the mean number of biopsies was greater when a trainee was not present, when a pediatric rather than an adult gastroenterologist performed the procedure, and when the patient was seen in an outpatient setting rather than as an inpatient.

More biopsies were performed when classic celiac disease endoscopic features were present, even though these features have a poor sensitivity and positive predictive value when applied to the dyspeptic population [16]. Moreover, the reliability of endoscopic features in celiac disease diagnosis has been disappointing [16]. In our study, $74 \%$ of patients with celiac disease had classic endoscopic features; however, about $70 \%$ of the PCD cohort did not have classic celiac disease features, which probably led to the lower number of biopsies obtained in this group. Therefore, biopsy number should not be influenced by gross examination. It is important to note, that an endoscopist might have a greater suspicion of celiac disease from gross appearance with newer diagnostic techniques [17], which might lead to a greater number of samples collected.

Patient demographic factors significantly influenced sample number. A higher index of suspicion, as reflected in the number of biopsy samples collected, diminished after the age of 60 years. This can be problematic because celiac disease can present after the age of 60 [18]. Our study demonstrated a similar reported pattern to that observed within the United States for biopsy disproportion with respect to ethnicity and race [19]. More biopsies were performed in Caucasian patients than in African American patients or other minority groups. This is likely to be due to a lower index of suspicion, as a lower prevalence of celiac disease has been reported in minority groups [20]. In our study, the suspicion for celiac disease in nonwhite patients was lower, leading to fewer biopsy samples. This suggests that celiac disease may be overlooked in minority groups [19].

In the examination of sex differences, the NCD cohort revealed a statistically significant discrepancy in biopsy number. This agrees with multiple epidemiological studies showing that women are more likely to be diagnosed [21]; however, serological screening reveals a more equal sex difference. Lebwohl et al. demonstrated that men are less likely to undergo duodenal biopsy than women for the same indication [19].

Manifestations of celiac disease such as chronic diarrhea, chronic anemia, or osteoporosis require outpatient evaluation. 
When patients present to the hospital and warrant endoscopic evaluation, their symptoms are typically more acute and severe. There were statistically significantly fewer samples obtained in the inpatient setting owing to a lower index of suspicion for celiac disease. However, fewer samples may have been obtained, as hospitalized patients are typically more ill and may have had clearer endoscopic signs requiring fewer samples, as biopsy would be targeted to the affected areas. The majority of celiac disease diagnoses were made in outpatients. Nonetheless, we cannot conclude that celiac disease should mainly be investigated in the outpatient setting because there was a disproportionate number of patients evaluated in this setting. Knowing the significant variation for celiac disease presentation and multiple disease associations that can be seen in a hospital setting, such as myocarditis, inflammatory bowel disease, sarcoidosis, IgA nephropathy, and epilepsy [22], it is essential that guidelines are adhered to regardless of the patient setting.

We delineated a group as PCD given the uncertainty regarding celiac disease among those with histological abnormalities that did not include villous atrophy with concomitant IEL. This group had significantly fewer biopsies. It is possible that some members of this group may have had celiac disease. The differential diagnosis of these more minor changes of increased IELs with normal villous height and villous abnormalities includes, as well as celiac disease, Helicobacter pylori-associated gastritis, medications (primarily nonsteroidal anti-inflammatory drugs), infections, bacterial overgrowth in the small intestine, and immune dysregulation [23]. Unless more specimens are evaluated, the pathologist could not confidently label patients with a definitive celiac disease diagnosis. The diagnosis of those labeled as PCD becomes challenging to manage, as a definitive therapeutic option cannot be offered for the patient's symptoms.

Classic celiac disease symptoms are diarrhea, steatorrhea, and weight loss due to malabsorption [3]. This notion is consistent with our observation: celiac disease was considered highly likely when the indication was diarrhea, as this indication had the greatest number of samples collected in the celiac disease cohort. Interestingly, it was nonspecific gastrointestinal symptoms and abdominal pain indications that had the most samples collected in the PCD cohort. Newer reports suggest that the clinical presentation of celiac disease for adults has changed over time, and typical presentation should not be expected in the adult population [24]. Thus, it becomes important to appropriately investigate the broader spectrum of symptoms and adhere to the guidelines irrespective of the indication.

Our initial suspicion was that the presence of a trainee would increase compliance with ACG recommendations. Previously, it has been demonstrated that the involvement of a trainee increased the detection rate of polyps during screening colonoscopies [25]. The results of this study did not support this hypothesis.

This study strongly demonstrates the importance of following guidelines in order to maximize the yield of celiac disease diagnosis during endoscopic evaluation. Following guidelines will also minimize the number of patients who are classified as PCD or who are misdiagnosed with no celiac disease. Previous studies demonstrated that a proportion of patients were diagnosed with celiac disease only after repeat EGD or in some instances multiple EGDs [1]. This reiterates the importance of collecting six samples. In addition to collecting the appropriate quantity of samples, targeting biopsies in the duodenal bulb, at the 9- or 12-o'clock position, may improve the yield [5]. It has been shown in several studies that celiac disease-related histological lesions are present in the bulb, with isolated mucosal abnormalities seen in up to $13 \%$ of celiac disease cases [26, 27]. Furthermore, histopathological abnormalities of celiac disease are patchy, and orientation of biopsy specimens can be variable, with crush biopsy artifacts $[13,15]$. Poor orientation and other technical processes render about $10.7 \%$ of biopsies inadequate [28]. The orientation of the specimen can be influenced by the number of bites during the forceps pass [29]. In addition, sample clumping, small biopsies, and incorrect preparation should be considered [30], as they may affect the final pathological reading. Interpretations of histological artifacts can be minimized by using the Corazza-Villanacci grading system rather than the Marsh criteria, to reduce the possible disagreement in gluten-sensitive enteropathy [31].

The strengths of our study include a large cohort examined over a 5-year time frame. The study was performed within a community setting, making the data clinically applicable, and multiple variables were simultaneously investigated. No previous studies have looked at the presence of a trainee or endoscopy clinical setting, and their impact on the number of samples collected. Limitations of our study include the retrospective design. Pertinent clinical data that would affect the pretest probability of detecting celiac disease, such as family history, human leukocyte antigen tissue transglutaminase antibody serology, and gluten-free diet response, were not incorporated, particularly in patients in the PCD cohort. Our finding of a higher yield of celiac disease diagnosis with higher specimen submission might be due to reverse causality, that is, patients who are most likely to have celiac disease may have more specimens submitted due to the underlying suspicion of celiac disease by the endoscopist. The post-EGD diagnosis (i.e. the final diagnosis in the NCD cohort) was not assessed, and the endoscopist may have been justified in not taking adequate samples for celiac disease if the definitive diagnosis was reached. Multiple pathologists were involved in the study, all of whom were general pathologists. PCD cases were not sent for outside expert pathology interpretation. Trainees from all three years of a fellowship program were included, and their degree of experience and grade of participation were not assessed. Furthermore, the location of the biopsy and the orientation within the duodenum were not assessed.

In summary, the yield of celiac disease diagnosis significantly improved with six biopsy samples. Adherence to guidelines when celiac disease is suspected is still not at goal, although there has been improvement in recent years. If celiac disease is considered in the differential diagnosis, it is critical that guidelines are followed to maximize the yield of the procedure. 
Competing interests

None

References

[1] Lebwohl B, Bhagat G, Markoff S et al. Prior endoscopy in patients with newly diagnosed celiac disease: a missed opportunity? Dig Dis Sci 2013; 58: $1293-1298$

[2] Brown IS, Smith J, Rosty C. Gastrointestinal pathology in celiac disease: a case series of 150 consecutive newly diagnosed patients. Am J Clin Pathol 2012; 138: $42-49$

[3] Rubio-tapia A, Hill ID, Kelly CP et al. ACG clinical guidelines: diagnosis and management of celiac disease. Am J Gastroenterol 2013; 108 : 656-676

[4] Rostom A, Murray JA, Kagnoff MF. American Gastroenterological Association (AGA) Institute technical review on the diagnosis and management of celiac disease. Gastroenterology 2006; 131: 1981-2002

[5] Kurien M, Evans KE, Hopper AD et al. Duodenal bulb biopsies for diagnosing adult celiac disease: is there an optimal biopsy site? Gastrointest Endosc 2012; 75: 1190 - 1196

[6] Pinto Sánchez MI, Smecuol E, Vázquez H et al. Very high rate of misdiagnosis of celiac disease in clinical practice. Acta Gastroenterol Latinoam 2009; 39: $250-253$

[7] Arguelles-grande C, Tennyson CA, Lewis SK et al. Variability in small bowel histopathology reporting between different pathology practice settings: impact on the diagnosis of coeliac disease. J Clin Pathol 2012; 65: 242-247

[8] Green PH, Murray JA. Routine duodenal biopsies to exclude celiac disease? Gastrointest Endosc 2003; 58: 92 - 95

[9] Cammarota G, Ianiro G, Sparano L et al. Image-enhanced endoscopy with I-scan technology for the evaluation of duodenal villous patterns. Dig Dis Sci 2013; 58: 1287 - 1292

[10] Rostami K, Kasturi R, Villanacci V et al. Challenges in endoscopy and histological diagnosis of celiac disease. Endoscopy 2011; 43: 375

[11] Lee SK, Green PH. Endoscopy in celiac disease. Curr Opin Gastroenterol 2005; 21: 589-594

[12] Antonioli DA. Celiac disease: a progress report. Mod Pathol 2003; 16: $342-346$

[13] Dickson BC, Streutker CJ, Chetty R. Coeliac disease: an update for pathologists. J Clin Pathol 2006; 59: 1008-1016

[14] Lebwohl B, Kapel RC, Neugut Al et al. Adherence to biopsy guidelines increases celiac disease diagnosis. Gastrointest Endosc 2011; 74: $103-109$

[15] Rostami-Nejad M, Villanacci V, Hogg-kollars S et al. Endoscopic and histological pitfalls in the diagnosis of celiac disease: a multicentre study assessing the current practice. Rev Esp Enferm Dig 2013; 105: $326-333$

[16] Dickey W, Hughes D. Disappointing sensitivity of endoscopic markers for villous atrophy in a high-risk population: implications for celiac disease diagnosis during routine endoscopy. Am J Gastroenterol 2001; 96: $2126-2128$

[17] Ianiro G, Gasbarrini A, Cammarota G. Endoscopic tools for the diagnosis and evaluation of celiac disease. World J Gastroenterol 2013; 19 : $8562-8570$

[18] Vilppula A, Collin P, Mäki M et al. Undetected coeliac disease in the elderly: a biopsy-proven population-based study. Dig Liver Dis 2008; 40: $809-813$

[19] Lebwohl B, Tennyson CA, Holub JL et al. Sex and racial disparities in duodenal biopsy to evaluate for celiac disease. Gastrointest Endosc 2012; 76: 779-785

[20] Brar P, Lee AR, Lewis SK et al. Celiac disease in African-Americans. Dig Dis Sci 2006; 51: $1012-1015$

[21] Murray JA, Van Dyke C, Plevak MF et al. Trends in the identification and clinical features of celiac disease in a North American community, 1950-2001. Clin Gastroenterol Hepatol 2003; 1: 19-27

[22] Reilly NR, Green PH. Epidemiology and clinical presentations of celiac disease. Semin Immunopathol 2012; 34: 473 - 478

[23] Bao F, Green PH, Bhagat G. An update on celiac disease histopathology and the road ahead. Arch Pathol Lab Med 2012; 136: 735 - 745

[24] Kav T, Sivri B. Is enteroscopy necessary for diagnosis of celiac disease? World J Gastroenterol 2012; 18: 4095-4101

[25] Buchner AM, Shahid MW, Heckman MG et al. Trainee participation is associated with increased small adenoma detection. Gastrointest Endosc 2011; 73: $1223-1231$

[26] Gonzalez S, Gupta A, Cheng J et al. Prospective study of the role of duodenal bulb biopsies in the diagnosis of celiac disease. Gastrointest Endosc 2010; 72: $758-765$

[27] Evans KE, Aziz I, Cross SS et al. A prospective study of duodenal bulb biopsy in newly diagnosed and established adult celiac disease. Am J Gastroenterol 2011; 106: 1837-1842

[28] Collin P, Kaukinen K, Vogelsang $\mathrm{H}$ et al. Antiendomysial and antihuman recombinant tissue transglutaminase antibodies in the diagnosis of coeliac disease: a biopsy-proven European multicentre study. Eur J Gastroenterol Hepatol 2005; 17: 85-91

[29] Latorre M, Lagana SM, Freedberg DE et al. Endoscopic biopsy technique in the diagnosis of celiac disease: one bite or two? Gastrointest Endosc 2015; 81: 1228-1233

[30] Ciacci C, De florio M. Evaluation of an instrument for the surveillance of adult gluten intolerance diagnosis: Report of the first year of activity of the Campania Celiac Network for Adult Celiac Disease. Dig Liver Dis 2007; 39: $703-704$

[31] Ravelli A, Villanacci V. Tricks of the trade: How to avoid histological pitfalls in celiac disease. Pathol Res Pract 2012; 208: 197-202 


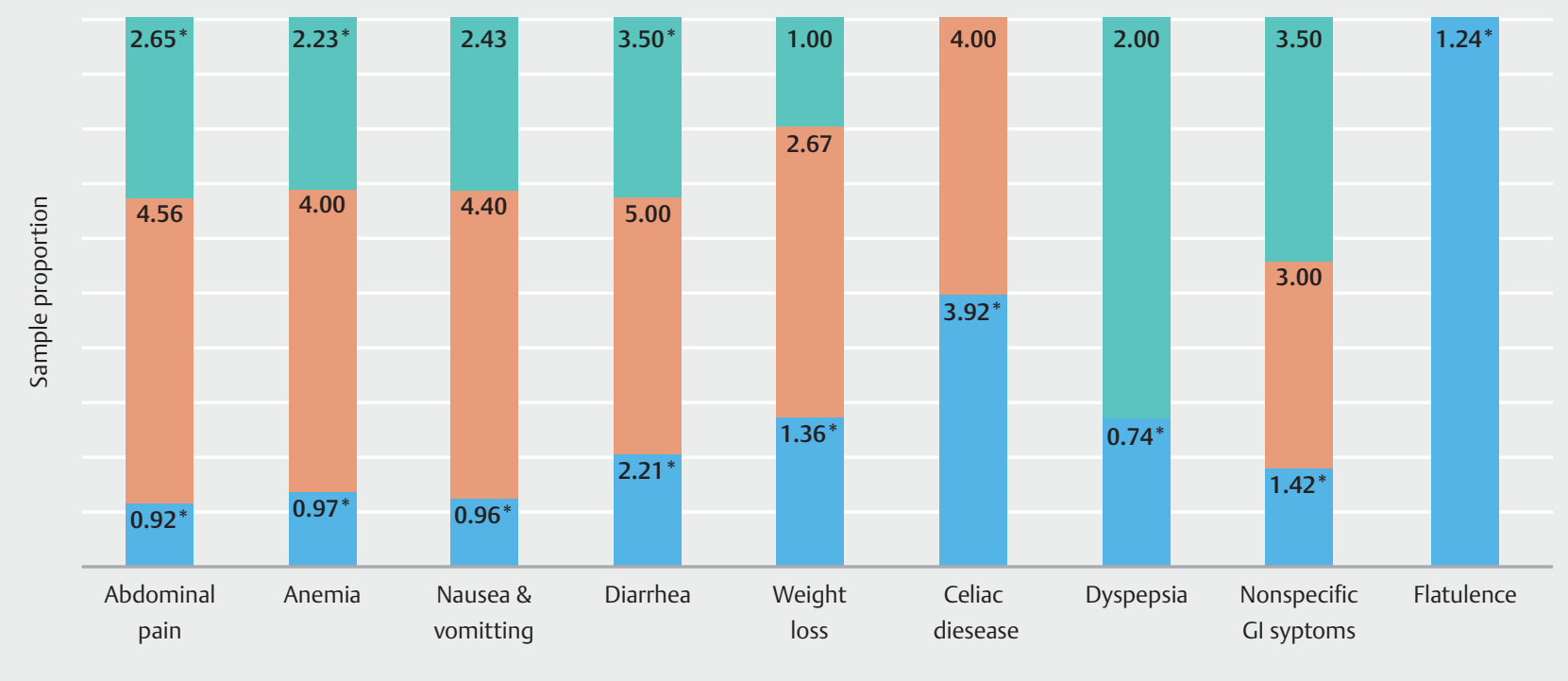

- Supplemental Fig. 1 Mean number of samples collected for each diagnosis cohort with regard to indication. PCD, possible celiac disease; CD, celiac disease; NCD, no celiac disease.

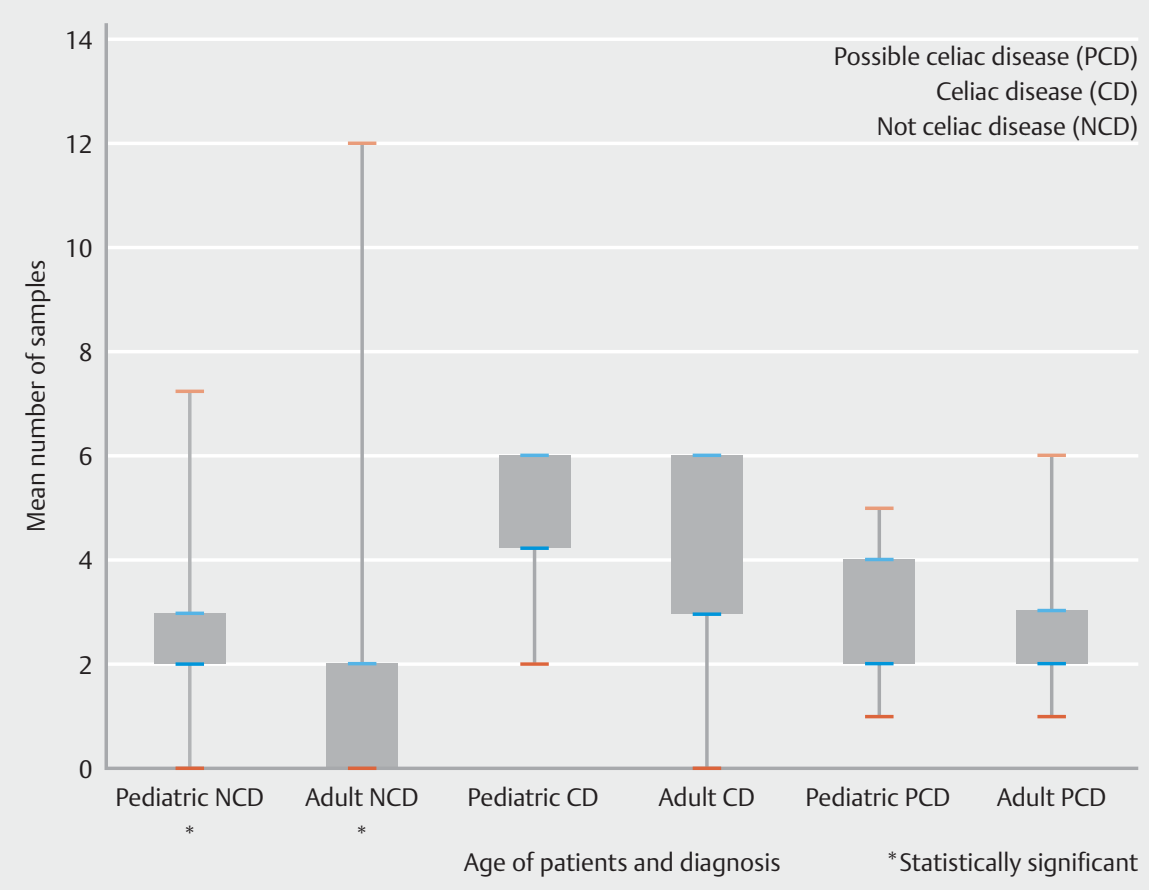

- Supplemental Fig. 2 A box-whisker plot illustrating the distribution of the mean number of samples collected for pediatric patients compared with adult patients for each diagnosis cohort. NCD, no celiac disease; CD, celiac disease PCD; possible celiac disease. 